\title{
A New Scoring: Can Brescia-COVID Respiratory Severity Scale Predict Mortality in Intensive Care?
}

\author{
(1) Burcu Illeri Fikri ${ }^{1}$, (1) Ezgi Direnç Yücel ${ }^{2}$, (1) Güldem Turan ${ }^{1}$
}

${ }^{1}$ University of Health Sciences Turkey, Başakşehir Çam and Sakura City Hospital, Intensive Care Unit, İstanbul, Turkey

${ }^{2}$ University of Health Sciences Turkey, Başakşehir Çam and Sakura City Hospital, Clinic of Anesthesiology and Reanimation, İstanbul, Turkey

\section{What is known on this subject?}

Intensive care mortality rates are estimating with acute physiology and chronic health evaluation II for many times. In coronavirus disease-2019 (COVID-19) pandemics, some new scales, algorithms and mortality scores were added to our practice.

\section{What this study adds?}

In this study, we aimed to use Brescia-COVID respiratory severity scale as a mortality predictor for COVID-19 related severe acute respiratory distress syndrome patients.

\section{ABSTRACT}

Objective: This study aims to compare the Brescia-coronavirus disease (COVID) severity scale (BCRSS) with acute physiology and chronic health evaluation II (APACHE-II) and sequential organ failure assessment (SOFA) scores in terms of predicting mortality in patients with severe coronavirus disease-2019 (COVID-19).

Material and Methods: BCRSS, SOFA, and APACHE-II scores of patients with severe COVID-19 were calculated when they were first admitted to the intensive care unit. BCRSS score calculation was repeated at the $48^{\text {th }}$ hour. Further treatment, intubation rates, and the result of the intensive care process were recorded and compared.

Results and Conclusion: When the three scoring systems are evaluated as the mortality indicators, SOFA score did not provide a statistically significant difference $(p>0.05)$, whereas the APACHE-II score was found to be significantly higher in the fatal cases $(p<0.01)$. Furthermore, BCRSS scores at the time of intensive care unit admission and at $48 \mathrm{~h}$ were significantly higher in the fatal cases $(p<0.01)$. As much as our experience with the disease has been increasing since the beginning of the pandemic, scoring systems are still used for patient triage area, intubation decisions, and directing the medical treatment. Although BCRSS, one of the COVID-19-specific scales, is yet to be validated, our results indicate its potential benefit for predicting IC mortality.

Keywords: Severe COVID-19, BCRSS, APACHE-II, SOFA, mortality, ICU

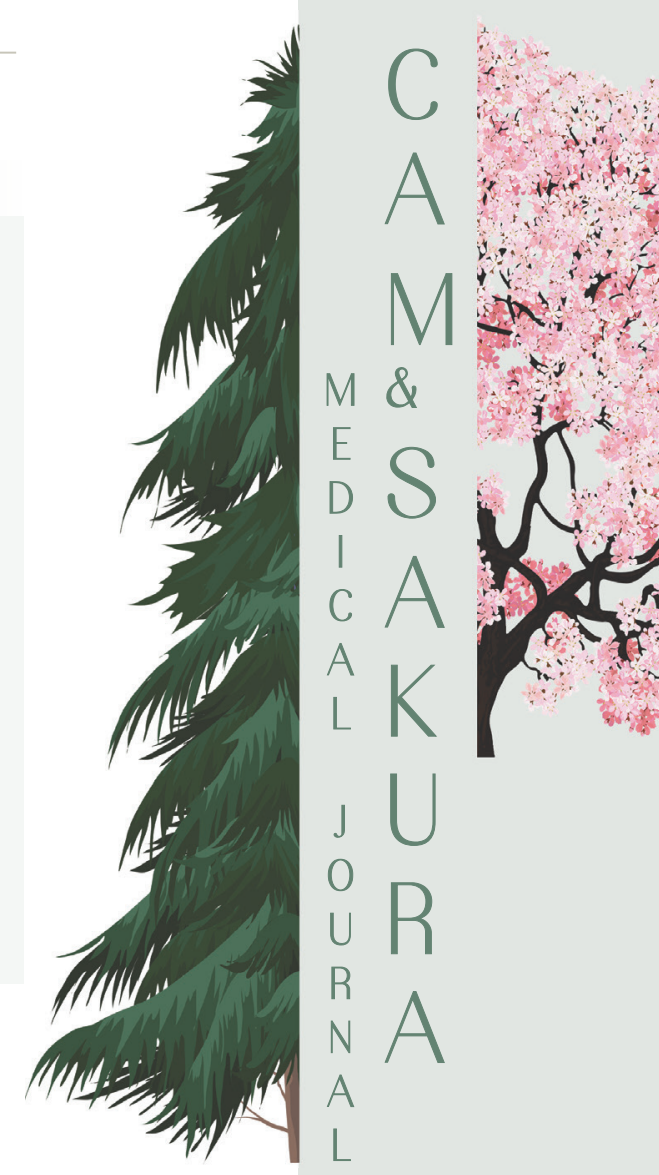

Address for Correspondence: Burcu Illeri Fikri MD, University of Health Sciences Turkey, Başakşehir Çam and Sakura City Hospital, Intensive Care Unit, İstanbul, Turkey

Phone: +90 2129096000 E-mail: drburcuileri@hotmail.com ORCID ID: orcid.org/0000-0002- 9220-5294 Received: 20.04.2021 Accepted: 14.08.2021

(C) Copyright 2021 by the Cam \& Sakura Medical Journal published by Galenos Publishing House. 


\section{Introduction}

The initial period of the coronavirus disease-2019 (COVID-19) pandemic caused significant anxiety in health workers not only due to a rapid increase in the number of patients, but also in terms of questions regarding in which units' patients will be treated, which treatments will be used, who will be intubated, and the right time for intubation. Unfortunately, as the large-scale pandemic continues with variant viruses throughout the world, rational and fair use of patient beds and airway equipment remains an important issue.

Italy being among the first countries to face the rapid increase in caseload, a fast and solid scaling/algorithm to be used for patient triage area and making invasive and noninvasive support decisions was needed in the Lombardy region. This need led to the Brescia-COVID severity scale (BCRSS) score (1). Although, BCRSS was first used to determine respiratory heaviness of the patients, and was utilized as a guide for patient management, it was also observed to be beneficial for decisions regarding the use of dexamethasone and tocilizumab. Despite the potential it exhibits, this scale still has a limited use since it is yet to be validated.

In this study, we aim to compare BCRSS score with acute physiology and chronic health evaluation II (APACHE-II), which we use for predicting the patient mortality, and with sequential organ failure assessment (SOFA) score, which we use as an illness severity indicator.

\section{Material and Methods}

We retrospectively scanned the Hospital Information Management System for the demographic variables, comorbid diseases, and laboratory and clinical data of 144 patients, who were admitted to our third level pandemic intensive care units (ICU) between January 01, 2021, and March 31, 2021. APACHE-II and SOFA score calculated within the first $24 \mathrm{~h}$ of ICU admission were recorded. BCRSS score was calculated based on the patient files and the data provided on ICU admissions, using BCRSS-calculator of the MD-Calc application. Intubation status (yes/no), laboratory results, medical treatments provided, and the result of ICU-care were also noted. This retrospective study was conducted with permission from the University of Health Sciences Turkey, Başakșehir Çam and Sakura City Hospital, Ethical Committee (2021.10.231).

\section{Statistical Analysis}

Statistical analyses were conducted with Number Cruncher Statistical System Statistical Software (Utah, USA). In addition to the descriptive statistics (mean, standard deviation, median, frequency, ratio, interquartile range), Shapiro-Wilk test and box plot graphics were used to assess if variables were normally distributed. Non-normally distributed variables were analyzed by Mann-Whitney $\mathrm{U}$ test, and in-group followups were compared with Wilcoxon signed-rank test. The relationship between the scores and mortality was evaluated with receiver operating characteristic (ROC) curve analysis, and values below the curve were compared using Binominal Exact test. Kaplan-Meier survival analysis and Log rank test was preferred for a survival analysis. Significance level was predetermined as $p<0.05$.

\section{Results}

The study was conducted with retrospective data of 144 patients, $38.9 \%$ female $(n=56)$ and $61.1 \%$ male $(n=88)$, who were hospitalized in a city hospital between January 1, 2021, and March 31, 2021. Age range of patients included in the study was between 26 and 91, with a mean of $64.63 \pm 11.76$.

Among the 144 patients, 118 died and 26 were discharged. A total of 128 patients were intubated, and 16 patients were observed in the ICU without intubation. Descriptive data, additional diseases, APACHE-II, SOFA, intubation status, result of the IC, and duration of IC stay are presented in Table 1.

For the 144 cases, mean APACHE-II score was 21.68 \pm 9.12 , mean SOFA score at ICU admission was $9.51 \pm 4.15$, BCRSS score at ICU admission was $4.72 \pm 1.76$, and BCRSS score in the $48^{\text {th }}$ hour was $6.53 \pm 1.88$.

Difference between the BCRSS scores was measured both at the admission and at the $48^{\text {th }}$ hour, and the significance levels are presented in Table 2.

The two-unit-difference between the BCRSS score on ICU admission (hour 0 ) and the $48^{\text {th }}$ hour was found to be statistically significant $(p<0.01)$.

When the BCRSS scores are evaluated on the basis of the treatment received specifically for severe COVID-19 symptoms, the change in the $48^{\text {th }}$ hour BCRSS score of the patients who did not receive pulse steroid treatment was statistically significant at the $p<0.05$ level, whereas it was at the $p<0.01$ level for patients who did receive the pulse treatment. BCRSS score between the two Anakinra subgroups was found to be significantly different in the $48^{\text {th }}$ hour, in comparison to the score on ICU admission $(p<0.01)$.

The change in the BCRSS score in the $48^{\text {th }}$ hour was found to be significantly different for the two Anakira subgroups $(p<0.01)$. BCRSS score was found to change significantly in the $48^{\text {th }}$ hour in patients who did not receive tocilizumab treatment $(p<0.01)$. This change was also significant for the 


\begin{tabular}{|c|c|c|}
\hline \multirow[t]{2}{*}{ Age } & Min-max (median) & $26-92(65)$ \\
\hline & Mean \pm SD & $64.63 \pm 11.76$ \\
\hline \multirow[t]{2}{*}{ Gender } & Female & $56(38.9)$ \\
\hline & Male & 88 (61.1) \\
\hline \multirow[t]{2}{*}{ Comorbidities } & No & $34(23.6)$ \\
\hline & Yes & $110(76.4)$ \\
\hline \multirow{10}{*}{ Diseases $(n=110)$} & Diabetes mellitus & $55(50.0)$ \\
\hline & Hypertension & 70 (63.6) \\
\hline & Hyperlipidemia & $2(1.8)$ \\
\hline & COPD & $17(15.5)$ \\
\hline & Malignancy & 15 (13.6) \\
\hline & CHF/ACS & $25(22.7)$ \\
\hline & Rheumatic disease & $1(0.9)$ \\
\hline & CVD & $6(5.5)$ \\
\hline & Dementia/Alzheimer's & $4(3.6)$ \\
\hline & Other & 35 (31.8) \\
\hline \multirow{2}{*}{ APACHE-II } & Min-max (median) & 7-48 (19.5) \\
\hline & Mean \pm SD & $21.68 \pm 9.17$ \\
\hline \multirow{2}{*}{ SOFA score } & Min-max (median) & $3-20(9)$ \\
\hline & Mean \pm SD & $9.51 \pm 4.15$ \\
\hline \multirow{2}{*}{ Intubation } & Yes & $128(88.9)$ \\
\hline & No & $16(11.1)$ \\
\hline \multirow{2}{*}{ Result } & Discharge & 26 (18.1) \\
\hline & Death & 118 (81.9) \\
\hline \multirow[t]{2}{*}{ Monitoring duration (days) } & Min-max (median) & $1-71(11)$ \\
\hline & $M \pm S D$ & $13.63 \pm 11.38$ \\
\hline
\end{tabular}

Table 2. Comparison of Brescia-COVID severity scale scores at the time of admission and the 48th hour

\begin{tabular}{|c|c|c|c|c|}
\hline & ICU admission & $48^{\text {th }}$ hour & Difference & \\
\hline & Median (IQR) & Median (IQR) & Median (IQR) & $\mathbf{p}$ \\
\hline BCRSS score & $4(3-6)$ & $7(6-8)$ & $2(0-4)$ & ${ }^{\mathrm{a}} 0.001^{* *}$ \\
\hline
\end{tabular}

patients who did receive tocilizumab treatment, but the significance level was $p<0.05$. As for the Plasmapheresis groups, the change in the $48^{\text {th }}$ hour BCRSS score was found to be significantly different between the patients who received and did not receive the treatment $(p<0.01)$. Similarly, when compared to the score on ICU admission, the change in the BCRSS scores in the $48^{\text {th }}$ hour was statistically different between the two intravenous immunoglobulin subgroups $(p<0.01)$. Evaluation of BCRSS scores based on the treatment subgroups is presented in detail in Table 3.
When the three scoring systems were evaluated as mortality indicators, SOFA score did not provide statistically significant results based on the mortality $(p>0.05)$ whereas APACHE-II score was significantly higher in patients who were deceased $(p<0.01)$. BCRSS scores on ICU admission and $48^{\text {th }}$ hour were significantly higher in the deceased cases $(p<0.01)$ (Table 4, 5, 6).

ROC Curve analyses of the scores are presented in Figure 1. 


\begin{tabular}{|c|c|c|c|c|c|}
\hline \multirow{3}{*}{$\begin{array}{l}\text { ICU admission } \\
\text { Median (IQR) }\end{array}$} & & \multicolumn{4}{|l|}{ BCRSS score } \\
\hline & & $48^{\text {th }}$ hour & \multicolumn{2}{|c|}{ Difference $(\Delta)$} & ${ }^{\mathrm{a}} \mathbf{p}$ \\
\hline & & Median (IQR) & \multicolumn{2}{|c|}{ Median (IQR) } & \\
\hline \multirow{3}{*}{ Pulse steroid } & No $(n=12)$ & $4(4-6)$ & $6(4.5-8)$ & $2(0-2)$ & $0.028^{*}$ \\
\hline & Yes $(n=132)$ & $4(3-6)$ & $7(6-8)$ & $2(0-4)$ & $0.001 * *$ \\
\hline & $\mathbf{p}$ & b0.887 & b0.173 & b0.552 & - \\
\hline \multirow{3}{*}{ Anakinra } & No $(n=104)$ & $4(3-6)$ & $7(6-8)$ & $2(0-4)$ & $0.001 * *$ \\
\hline & Yes $(n=40)$ & $4(3-6)$ & $7(6.5-8)$ & $2.5(0-4)$ & $0.001 * *$ \\
\hline & p & ${ }^{\mathrm{b}} 0.472$ & ${ }^{\mathrm{b}} 0.450$ & ${ }^{\mathrm{b}} 0.064$ & - \\
\hline \multirow{3}{*}{ Tocilizumab } & No $(n=130)$ & $4(3-6)$ & $7(6-8)$ & $2(0-4)$ & $0.001 * *$ \\
\hline & Yes $(n=14)$ & $4.5(4-7)$ & $8(6-8)$ & $0.5(0-2)$ & $0.048^{*}$ \\
\hline & $p$ & ${ }^{\mathrm{b}} 0.182$ & b0.816 & ${ }^{\mathrm{b}} 0.238$ & - \\
\hline \multirow{3}{*}{ Plasmapheresis } & No $(n=124)$ & $4(3-6)$ & $7(5.5-8)$ & $2(0-4)$ & $0.001 * *$ \\
\hline & Yes $(n=20)$ & $4.5(4-6)$ & $8(7-8)$ & $2(0-4)$ & $0.001 * *$ \\
\hline & p & ${ }^{\mathrm{b}} 0.256$ & ${ }^{\mathrm{b}} 0.178$ & ${ }^{\mathrm{b}} 0.384$ & - \\
\hline \multirow{3}{*}{ IVIG } & No $(n=118)$ & $4(3-6)$ & $7(6-8)$ & $2(0-4)$ & $0.001 * *$ \\
\hline & Yes $(n=26)$ & $4(4-6)$ & $8(6-8)$ & $2.5(0-4)$ & $0.001 * *$ \\
\hline & $p$ & b0.735 & ${ }^{\mathrm{b}} 0.075$ & ${ }^{\mathrm{b}} 0.076$ & - \\
\hline
\end{tabular}

\begin{tabular}{|c|c|c|c|c|}
\hline & & Survive & Exitus & p \\
\hline \multirow{2}{*}{ SOFA } & Mean $\pm S D$ & $8.12 \pm 2.52$ & $9.82 \pm 4.38$ & \multirow{2}{*}{0.116} \\
\hline & Median (min-max) & $8(3-12)$ & $9(3-20)$ & \\
\hline \multirow{2}{*}{ APACHE-II } & Mean \pm SD & $14.81 \pm 5.37$ & $23.19 \pm 9.15$ & \multirow{2}{*}{$0.001 * *$} \\
\hline & Median (min-max) & $14(8-30)$ & $22(7-48)$ & \\
\hline \multirow{2}{*}{ BCRSS on ICU admission } & Mean \pm SD & $3.65 \pm 1.16$ & $4.96 \pm 1.78$ & \multirow{2}{*}{$0.001 * *$} \\
\hline & Median (min-max) & $3(2-7)$ & $4(2-8)$ & \\
\hline \multirow{2}{*}{ BCRSS in $48^{\text {th }}$ hour } & Mean \pm SD & $3.88 \pm 1.99$ & $7.11 \pm 1.27$ & \multirow{2}{*}{$0.001 * *$} \\
\hline & Median (min-max) & $3(0-8)$ & $8(3-8)$ & \\
\hline
\end{tabular}

\begin{tabular}{|c|c|c|c|c|c|}
\hline \multicolumn{6}{|l|}{ Area under the curve } \\
\hline \multirow[t]{2}{*}{ Test result variable(s) } & \multirow[t]{2}{*}{ Area } & \multirow[t]{2}{*}{ Standard error ${ }^{\mathrm{a}}$} & \multirow[t]{2}{*}{ Asymptotic $p^{b}$} & \multicolumn{2}{|c|}{ Asymptotic $95 \%$ confidence interval } \\
\hline & & & & Lower bound & Upper bound \\
\hline SOFA score & 0.598 & 0.052 & 0.117 & 0.496 & 0.700 \\
\hline APACHE-II & 0.792 & 0.046 & $0.000 * *$ & 0.701 & 0.882 \\
\hline BCRSS on ICU admission & 0.723 & 0.051 & $0.000 * *$ & 0.623 & 0.824 \\
\hline BCRSS in $48^{\text {th }}$ hour & 0.891 & 0.042 & $0.000 * *$ & 0.809 & 0.973 \\
\hline
\end{tabular}




\begin{tabular}{|c|c|}
\hline Dual comparison of areas & $\mathbf{p}$ \\
\hline SOFA-APACHE-II & $0.005^{* *}$ \\
\hline SOFA-BCRSS on ICU admission & 0.102 \\
\hline SOFA-BCRSS $48^{\text {th }}$ hour & $<0.001 * *$ \\
\hline APACHE-II- BCRSS on ICU admission & 0.206 \\
\hline APACHE-II- BCRSS in $48^{\text {th }}$ hour & $0.021 *$ \\
\hline $\begin{array}{l}\text { BCRSS on ICU admission - BCRSS in } \\
48^{\text {th }} \text { hour }\end{array}$ & $0.002^{* *}$ \\
\hline \multicolumn{2}{|c|}{$\begin{array}{l}\text { Binomial Exact test, } * * 0<0.01 \text {, SOFA: Sequential organ failure assessment, } \\
\text { APACHE-II: Acute physiology and chronic health evaluation II, BCRSS: } \\
\text { Brescia-COVID severity scale, ICU: Intensive care units }\end{array}$} \\
\hline
\end{tabular}

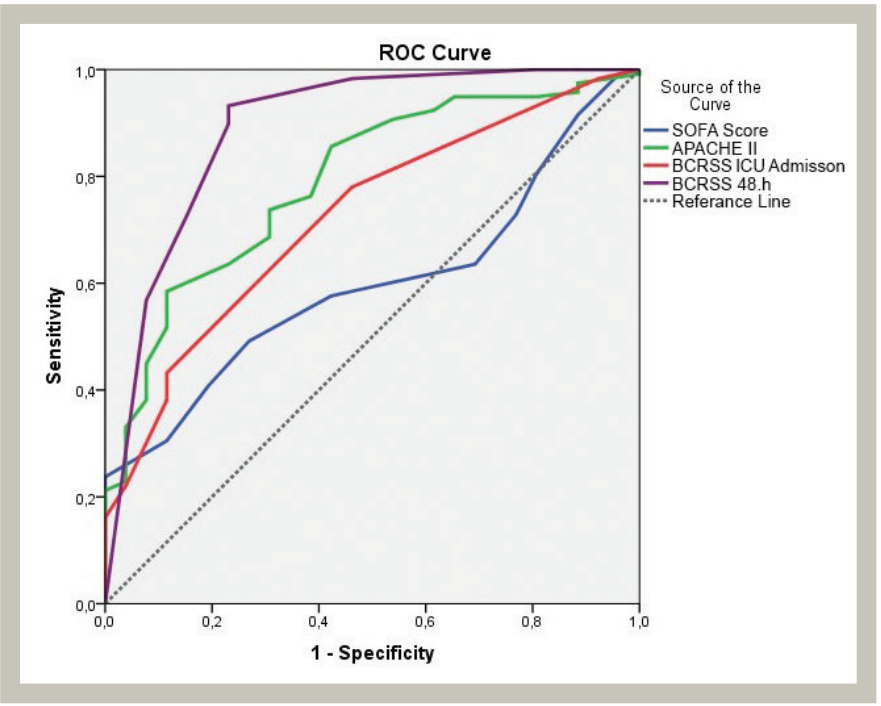

Figure 1. Receiver operating characteristic curves of SOFA, APACHEII, and BCRSS

ROC: Receiver operating characteristic curves, SOFA: Sequential organ failure assessment, APACHE-II: Acute physiology and chronic health evaluation II, BCRSS: Brescia-COVID severity scale

\section{Discussion}

Rational management of ICUs has gained significant importance, beginning with the COVID-19 pandemic. As a result, new additions are being made to our scoring systems, which we use for ICU admissions, treatment management, and illness severity prediction. Scoring systems extensively used in ICUs are "prognostic scoring systems," which predict mortality, and "organ failure scoring systems," which evaluate morbidity. The most frequently used prognostic scoring systems are APACHE, simplified acute physiology score (SAPS), therapeutic intervention scoring system, and mortality prediction/probability models. Furthermore, SOFA, multiple organ dysfunction score, and logistic organ dysfunction score are some of the widely accepted organ failure scoring systems
$(2,3,4)$. APACHE-II, SAPS-II, SOFA scores could be calculated electronically in our hospital's information system.

At the beginning of the pandemic, BCRSS scoring system and quick COVID-19 severity index (qCSI) were developed for managing triage in the Lombardia region, where some of the first cases were observed. In our hospital, we still prioritize APACHE-II as an objective indicator for evaluating the possibility of mortality and illness severity during ICU admissions. Siddiqi and Mehra (5) define three stages in the diagnosis and treatment of COVID-19-related illnesses: Mild, moderate, and severe. According to this classification, patients included in our study were all in stage 3 (severe) since po2/fio2 was $<300$ for each one of them. In 144 patients, 128 were intubated and monitored during invasive mechanical ventilation.

Due to its significance in the diagnosis of sepsis and septic shock, SOFA is a scoring system that we use daily or, based on the clinician's preference, even more frequently could be used. The literature on SOFA scores of patients with COVID-19 indicate its potential use for predicting mortality. Rod and colleagues list 60 independent predictors for predicting the severity of COVID-19, and report that SOFA, age, D-dimer, high-sensitive C-reactive protein, body temperature, albumin level, and presence of comorbidity (e.g., diabetes mellitus) are highly related to illness severity (6). In the same study, authors suggest that SOFA score could be used as a parameter for hospital mortality prediction. Kodik et al. (7) investigated SOFA and some other mortality evaluation criteria, including subgroups of SOFA parameters, and reported that SOFA score could be used for mortality prediction. On the other hand, Raschke et al. (8) have conducted another study, which indicates low discriminative performance of SOFA score in mortality prediction, which was lower than the age factor alone. SOFA score uses six parameters to evaluate the six systems. However, for COVID-19, mostly three organ systems (respiratory, hepatobiliary, and renal) were found to be mortality-related (9). In our study, median value for the SOFA score was nine, and our results did not indicate a significant prediction performance of SOFA on mortality. As the previous studies have suggested, this may be due to the sepsis-specific design of SOFA score, which does not include mortality increasing parameters in COVID-19, such as age or comorbidity. Furthermore, as a limitation in our study, SOFA score was calculated only once within $48 \mathrm{~h}$ of ICU admission, and therefore further SOFA scores of patients were not included in the analyses.

APACHE-II is another scoring system used for mortality prediction within the first $24 \mathrm{~h}$ of ICU admission, together with 
the presenting symptoms, laboratory results, and presence of additional acute and chronic diseases. The score ranges from 0 to 71, higher numbers indicating stronger expectations of mortality (10). Zou et al. (11) conducted a study with patients with COVID-19 and suggested that APACHE-II scores $>17$ are alarming for mortality and should be considered in treatment decisions. Chen et al. (12) evaluated the severity and mortality of COVID-19 pneumonia using APACHE-II, CURB-65, and pneumonia severity index, and reported all three scales as the viable options. In parallel, our results indicate a relationship between high APACHE-II scores and high mortality. Our APACHE-II median value was 19.5 (minimum-maximum 7-48), and 118 of the 144 patients did not survive. The median APACHE-II score of surviving patients was 14, whereas it was 22 for the exitus group. This was an expected result which could be explained by the severity of our patient group, the development of multiple organ failure, the high mean age $(60+)$, and the high number of patients with comorbidity.

BCRSS provides a gradual approach to the management of patients with validated or predicted COVID-19 pneumonia. BCRSS is used for patients who present with COVID-19 pneumonia or describe symptoms going back $>7$ days. In these patients, four criteria are evaluated, and the algorithm presented suggestions based on the presence of $\leq 2$ or $>2$ criteria. According to the algorithm, if more than two criteria are present, high-flow oxygen treatment (HFOT) or noninvasive mechanical ventilator (NIMV) are suggested. If more than two criteria are positive despite the NIMV and/or HFOT support, considering the age and comorbidities of the patient, decision to intubate may be made. Suggestions provided by the algorithm consist of eight layers. With every change in the patient's status and every new treatment provided, calculations could be remade, making BCRSS a dynamic and timely scale.

Even though BCRSS was, at first, used for determining the respiratory severity and guiding the patient management, it was also found to be useful for making decisions in two other areas (i.e., dexamethasone and tocilizumab treatments). Italian working group suggests treating patients with a BCRSS score of/higher than two with dexamethasone (13). Similarly, treating patients who have a BCRSS score of/higher than three with tocilizumab is suggested. The BCRSS score is based on an algorithm that provides a guide for many patient management issues, including the invasive/noninvasive respiratory support, prone positioning, treatment agents, and laboratory test orders. In Italy, in general, treatment decisions in emergency rooms, hospital services, and ICU were made through this scoring system, utilized by the clinician as frequently as preferred. For patients with COVID-19 pneumonia, for patients who describe symptoms going back at least seven days, and for patients who are polymerase chain reaction (PCR) (+) or whose PCR is inconclusive, all four test criteria are evaluated. These test criteria are applied on the algorithm, leading to a score from 0 to 8 . The algorithm makes a treatment suggestion based on the score calculated. The scale was designed to be dynamic, to be consulted frequently and to provide new scores after each treatment (1). If the score is $\geq 4$, the need for ICU admission and intubation should be considered. This scale, despite its apparent convenience, still has limited use since it is yet to be validated. However, the studies are being conducted for evaluating the use of BCRSS scores. For example, Ak et al. (14) analyzed ICU admission and mortality rates of all patients hospitalized with COVID-19 diagnosis, using BCRSS and qCSI. Authors report both scales to be viable options for this purpose (14). Similarly, Rodriguez-Nava et al. (15) compared different scales in terms of ICU admission and mortality prediction and suggest qCSI and BCRSS to be good indicators in this area. In parallel to this, in our study, BCRSS median value on ICU admission was four, and the BCRSS median value in the $48^{\text {th }}$ hour was seven. The difference between the two values was statistically significant in terms of the mortality rates. The gradual scoring in BCRSS algorithm could be a useful guide for clinicians. Studies suggest that BCRSS could be used for making tocilizumab and Anakinra treatment choices $(16,17)$. In our study, dexamethasone and methylprednisolone treatment is applied in pandemic services and intermediate care units, where patients are treated before ICU admission. There are studies suggesting a BCRSS score of three to be an indicator for evaluating the dexamethasone option (18). In our study, the majority of the patients received $1 \mathrm{mg} /$ $\mathrm{kg} /$ day methylprednisolone or pulse methylprednisolone (250 mg/day for 3 days), and their treatment was continued with the same dosage of steroid or an increased dosage of pulse steroid (250-1,000 mg/day). It could be argued that, for patients in services, this scoring system could be used for determining the need for steroid treatment. Italian society of Infectious and tropical diseases suggests a BCRSS score of $\geq 3$ for tocilizumab treatment (13). Based on this suggestion, Erden et al. (19) designed a study to compare BCRSS and other scales for Anakinra treatment decisions and reported the superiority of BCRSS, SOFA, and MuLBSTA scores to the H-score in the development of macrophage activation syndrome (19). In our study, we used BCRSS scores for predicting mortality. According to our results, BCRSS score calculated in the $48^{\text {th }}$ hour was the best predictor.

\section{Study Limitations}

As limitations of our study, the design did not include a control group, and we haven't used BCRSS to apply steroid and 
anti-cytokine treatments. The required laboratory tests for recalculating the SOFA scores were not fully ordered, resulting in a lack of data for evaluating daily increases/decreases in the SOFA score.

\section{Conclusion}

To conclude; our evaluations indicate that as the COVID-19 pandemic has been present for more than two years and as the patients still present severe symptoms due to additional variant viruses, the need for valid scoring systems will persist for not only triage purposes but also for the rational use of ventilators and ICU beds as well as for predicting the mortality. BCRSS score, specifically designed for COVID-19, is still not validated probably since the algorithm has yet not been tested on significant number of patients. Further studies may contribute to the validation of BCRSS for more reliable results.

\section{Ethics}

Ethics Committee Approval: University of Health Sciences Turkey, Başakșehir Çam and Sakura City Hospital, Ethical Committee (2021.10.231).

Informed Consent: Retrospective study.

Peer-review: Externally peer-reviewed.

\section{Authorship Contributions}

Surgical and Medical Practices: B.I.F., E.D.Y., Concept: B.I.F., G.T., Design: B.I.F., G.T., Data Collection or Processing: B.I.F., E.D.Y., Analysis or Interpretation: B.I.F., G.T., Literature Search: B.I.F., G.T., Writing: B.I.F., G.T.

Conflict of Interest: No conflict of interest was declared by the authors.

Financial Disclosure: The authors declared that this study received no financial support.

\section{REFERENCES}

1. Duca A, Piva S, Focà E, Latronico N, Rizzi M. Calculated decisions: Brescia-COVID respiratory severity scale (BCRSS)/algorithm. Emerg Med Pract 2020;22(Suppl 5):CD1-CD2.

2. Vincent JL, Moreno R. Clinical review: scoring systems in the critically ill. Crit Care 2010;14:207.

3. Strand K, Flaatten H. Severity scoring in the ICU: a review. Acta Anaesthesiol Scand 2008;52:467-478.

4. Keegan MT, Gajic O, Afessa B. Severity of illness scoring systems in the intensive care unit. Crit Care Med 2011;39:163-169.

5. Siddiqi HK, Mehra MR. COVID-19 illness in native and immunosuppressed states: a clinical-therapeutic staging proposal. J Heart Lung Transplant 2020;39:405-407.

6. Rod JE, Oviedo-Trespalacios 0, Cortes-Ramirez J. A brief-review of the risk factors for covid-19 severity. Rev Saude Publica 2020;54:60.

7. Kodik MS, Öztürk E, Uz I, Özçete E, İnci Ö, Ersel M. Evaluation of mortality in ICU-hospitalized COVID-19 patients by using REMS, APACHE-II, CCI, and SOFA. Research Square 2021. doi: 10.21203/ rs.3.rs641424/vl.

8. Raschke RA, Agarwal S, Rangan P, Heise CW, Curry SC. Discriminant accuracy of the SOFA score for determining the probable mortality of patients with COVID-19 pneumonia requiring mechanical ventilation. JAMA 2021;325:1469-1470.

9. Gupta S, Hayek SS, Wang W, et al. STOP-COVID Investigators. Factors associated with death in critically ill patients with coronavirus disease 2019 in the US. JAMA Intern Med 2020;180:1436-1446.

10. Naved SA, Siddiqui S, Khan FH. APACHE-II score correlation with mortality and length of stay in an intensive care unit. J Coll Physicians Surg Pak 2011;21:4-8.

11. Zou X, Li S, Fang M, et al. Acute physiology and chronic health evaluation II score as a predictor of hospital mortality in patients of coronavirus disease 2019. Crit Care Med 2020;48:e657-e665.
12. Chen J, Liu B, Du H, et al. Performance of CURB-65, PSI, and APACHE-11 for predicting COVID-19 pneumonia severity and mortality. Eur J Inflamm 2021. doi:10.1177/20587392211027083.

13. Lombardy Section Italian Society Infectious and Tropical Diseases. Vademecum for the treatment of people with COVID-19. Edition 2.0, 13 March 2020. Infez Med 2020;28:143-152.

14. Ak R, Kurt E, Bahadirli S. Comparison of 2 risk prediction models specific for COVID-19: the Brescia-COVID respiratory severity scale versus the quick COVID-19 severity index. Disaster Med Public Health Prep 2021;15:e46-e50.

15. Rodriguez-Nava G, Yanez-Bello MA, Trelles-Garcia DP, Chung CW, Friedman HJ, Hines DW. Performance of the quick COVID-19 severity index and the Brescia-COVID respiratory severity scale in hospitalized patients with COVID-19 in a community hospital setting. Int J Infect Dis 2021;102:571-576.

16. Moreno-Pérez O, Andres M, Leon-Ramirez JM, et al. Experience with tocilizumab in severe COVID-19 pneumonia after 80 days of followup: a retrospective cohort study. J Autoimmun 2020;114:102523.

17. Toniati P, Piva S, Cattalini M, et al. Tocilizumab tor the treatment of severe COVID-19 pneumonia with hyperinflammatory syndrome and acute respiratory failure: a single center study of 100 patients in Brescia, Italy. Autoimmun Rev 2020;19:102568.

18. Pennica A, Conforti G, Falangone F, Martocchia A et al. clinical management of adult coronavirus infection disease 2019 (COVID-19) positive in the setting of low and medium intensity of care: a short practical review. SN Compr Clin Medi 2020;2:694-699.

19. Erden A, Ozdemir B, Karakas O, et al. Evaluation of 17 patients with COVID-19 pneumonia treated with anakinra according to HScore, SOFA, MULBSTA, and Brescia-COVID respiratory severity scale (BCRSS) scoring systems. J Med Virol 2021;93:1532-1537. 